\title{
Quantum jumps and spin dynamics of interacting atoms in a strongly coupled atom-cavity system
}

\author{
M. Khudaverdyan ** W. Alt, T. Kampschulte, S. Reick, A. Thobe, A. Widera, and D. Meschede \\ Institut für Angewandte Physik, Universität Bonn, Wegelerstr. 8, 53115 Bonn, Germany
}

\begin{abstract}
We experimentally investigate the spin dynamics of one and two neutral atoms strongly coupled to a high finesse optical cavity. We observe quantum jumps between hyperfine ground states of a single atom. The interaction-induced normal mode splitting of the atom-cavity system is measured via the atomic excitation. Moreover, we observe the mutual influence of two atoms simultaneously coupled to the cavity mode.
\end{abstract}

PACS numbers: $37.30 .+\mathrm{i}, 42.50 . \mathrm{Lc}, 42.50 . \mathrm{Pq}$

The strong coherent light-matter interaction induced by a high finesse resonator allows to study and manipulate neutral atoms at the quantum level [1. Information about the evolution of the coupled system is contained in both the resonator light field and the atom. In particular, the dynamics of a single atom coupled to the cavity can be inferred with a high information rate from the light field. Continuous monitoring of the atomic motion and the atom number have already been demonstrated [2, 3, 4]. However, the dynamics of the internal spin states, which plays a central role in many proposals to engineer entangled quantum states [5, 6], has so far not been resolved.

Here we observe the internal dynamics of one and two atoms in a high finesse optical resonator. Continuous observation of a quantum system can reveal quantum jumps, i.e. the sudden and random change of a quantum system's state over time due to interaction with the environment, as has been observed in various systems [7, 8, 9, 10. Here we use the cavity to directly observe the spin quantum jumps of a single atom, as anticipated in Ref. [4. This method is based on the suppression of the cavity transmission [11] due to strong coherent interaction, leading to normal mode splitting. For single atoms this splitting has been observed by measuring the intra-cavity photon number 12,13 . In contrast, we use the cavity-based detection of the atomic state to measure the normal mode splitting via the atomic part of the excitation. Extending our experiment to the simultaneous coupling of two atoms to the cavity mode we observe the coupled dynamics of the atomic states, as the state of each atom influences the intra-cavity photon number experienced by the other atom.

In our approach neutral Caesium (Cs) atoms are coupled to the mode of an optical resonator as depicted in Fig. 11. We monitor the transmission of a probe laser beam with angular frequency $\omega_{\mathrm{p}}$, tuned to the resonance frequency of the cavity $\omega_{\mathrm{c}}=\omega_{\mathrm{p}}$. The cavity itself is blue-

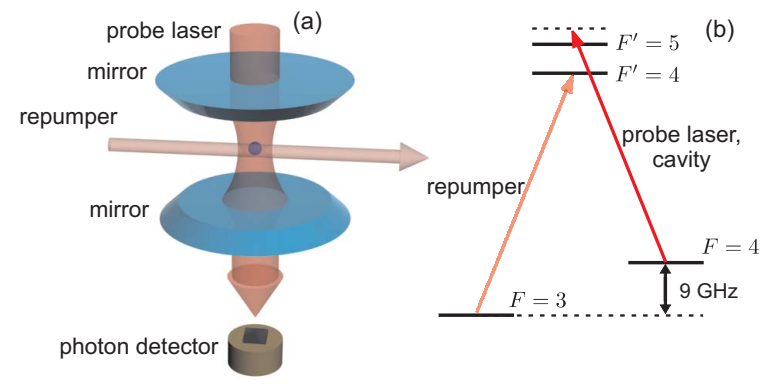

FIG. 1: (a) A single atom is placed into the cavity mode. The state of the atom is continuously monitored by observing the transmission of the probe laser, tuned close to the $F=4 \longrightarrow F^{\prime}=5$ transition. A similar scheme applies to two atoms. (b) Level scheme of relevant energy levels and transitions for Cs.

detuned by $\omega_{\mathrm{c}}-\omega_{4,5^{\prime}}=2 \pi \times 44 \mathrm{MHz}$ from the $F=4 \longrightarrow F^{\prime}=5$ transition of the Cs $D_{2}$ line at $852 \mathrm{~nm}$ [14], see Fig. 1]. Here $\omega_{4,5^{\prime}}$ is the angular frequency of the $F=4 \longrightarrow F^{\prime}=5$ transition, where $F$ is the total atomic angular momentum. The cavity transmission level is a direct and continuous measure of the atomic state: An atom in the $F=3$ hyperfine state does not couple to the resonator mode, hence the laser beam is fully transmitted. An atom in the $F=4$ state, however, couples strongly to the cavity mode and leads to a normal-mode splitting of the cavity resonance. This effectively blocks the transmission of the probe laser beam.

At the beginning of each experimental sequence we load a single laser cooled Cs atom into a standing-wave optical dipole trap at $1030 \mathrm{~nm}$. Using the trap as an optical conveyor belt we transport the atom to a well defined position within the field of the cavity, for details see [15. The cavity mode has a diameter of $2 \omega_{0}=46 \mu \mathrm{m}$ and a length of $159 \mu \mathrm{m}$. The parameters of our atom-cavity system are $\{g, \kappa, \gamma\}=2 \pi \times\{8 \ldots 13,0.4,2.6\} \mathrm{MHz}$. The expected atom-cavity coupling strength $g$ varies for different Zeeman sublevels of the $F=$ 


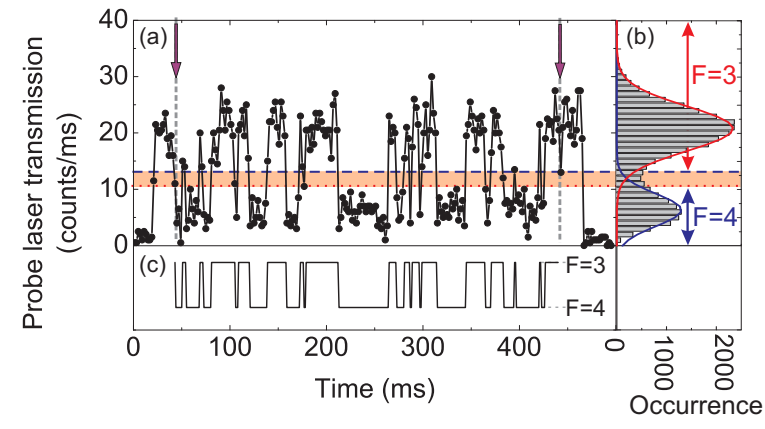

FIG. 2: (a) Measured cavity transmission over time, exhibiting quantum jumps of a single atom between $F=3$ and $F=4$. Arrows indicate the insertion and removal of the atom. (b) Histogram of the transmission levels. The two peaks represent the $F=3$ and $F=4$ states, the horizontal dashed (dotted) line marks the thresholds for the spin $F=3(F=4)$ state (see text). (c) Reconstructed atomic state for the transmission signal in (a).

$4 \longrightarrow F^{\prime}=5$ transition, $2 \gamma$ and $2 \kappa$ are the linewidths of the $D_{2}$ transition and cavity resonance, respectively. Thus our experiment operates in the strong coupling regime with a single atom cooperativity parameter $C_{1}=g^{2} /(2 \kappa \gamma)>30$.

When we observe the cavity transmission continuously, a random telegraph signal originates from quantum jumps of a single atom between the $F=4$ (low transmission signal) and $F=3$ (high transmission signal) hyperfine states over about $400 \mathrm{~ms}$ (see Fig. 2a). The intra-cavity photon number for high transmission is equal to 0.3 . With an overall photon detection efficiency of $1.3 \%$ the resulting count rate at the photon detector is about 20 counts $/ \mathrm{ms}$. The transitions from the $F=4$ to the $F=3$ state are caused by the cavity field, offresonantly exciting the atom to the $F^{\prime}=4$ state, from which it decays into the $F=3$ ground state. For atoms in the $F=3$ state the rate of quantum jumps is strongly reduced due to the large detuning of the probe laser from the $F=3 \longrightarrow F^{\prime}=4$ transition. To induce transitions from the $F=3$ to the $F=4$ state at a comparable rate, we apply an additional repumping laser which is resonant with the $F=3 \longrightarrow F^{\prime}=4$ transition (see Fig. 1). Although strong repumping lasers have been applied in previous work with single atoms [3, 11, for our repump intensity quantum jumps can be resolved with our time resolution of $2 \mathrm{~ms}$, given by the binning time.

In order to extract the atomic state from the transmission signal we form a histogram from 163 telegraph traces (see Fig. 2(b)) which shows two peaks, reflecting the $F=3$ and $F=4$ spin states, respectively. We fit each peak with a Gaussian dis- tribution. The two peaks partially overlap, so that in this range the spin state cannot be determined unambiguously. We therefore define the region of transmission values corresponding to the $F=4$ state, denoted in Fig. 2 (b) by the blue arrow, such that only $1 \%$ of the values for an atom in $F=3$ lies in this region. Vice versa, the region labeled by the red arrow is chosen to contain only $1 \%$ of the transmission values related to the $F=4$ state and is therefore used to indicate $F=3$. In the overlap region, which contains about $4 \%$ of all time bins, the ambiguity is resolved if the previous and subsequent transmission values correspond to the same spin state. In this case the middle value is considered as noise, as it would otherwise correspond to two directly subsequent quantum jumps which are much less probable. If previous and subsequent values are different, the middle value in question is interpreted as a quantum jump. This allows us to reconstruct the atomic state, see Fig. 2 (c).

We characterize the dynamics of the quantum jumps by analyzing the second order correlation function of the reconstructed atomic state as proposed in Ref. [17, and thereby determine the rates of the quantum jumps. From the correlation function we find the rate for a quantum jump from $F=4$ to $F=3$ to be about $106 \mathrm{~s}^{-1}$, set by the intensity of the probe laser field inside the cavity. Likewise, the rate of jumps from $F=3$ to $F=4$ is found to be $42 \mathrm{~s}^{-1}$, corresponding to the applied repumping laser intensity.

We use this atomic spin detection method to deduce the normal mode splitting from the decay of the atomic excitation, rather than the photonic excitation. In the first step of the measurement, we induce the normal mode splitting by coupling an atom in the $F=4, m_{F}=4$ state to the resonator, this time tuned close to the $F=4 \longrightarrow F^{\prime}=4$ transition. Here, $m_{F}=4$ is the projection of the angular momentum onto the quantization axis. The atom-cavity system is then probed with a weak $70 \mu$ s probe laser pulse with variable detuning $\omega_{\mathrm{p}}-\omega_{\mathrm{c}}$, adjusted to not saturate the population transfer to $F=3$. If the atom gets excited to the $F^{\prime}=4$ state, it can decay to the $F=3$ and $F=4$ ground states with comparable probabilities. Consequently, the atomic excitation probability is mapped onto the population of the dark $F=3$ ground state. Remarkably, during this process the atom scatters on average only two photons, therefore experiencing negligible heating as it decouples from the cavity in the $F=3$ state. In a second step, we shift the cavity resonance $40 \mathrm{MHz}$ to the blue of the $F=4 \longrightarrow F^{\prime}=5$ transition and record the transmission of the resonant probe laser. From the first $2 \mathrm{~ms}$ of the transmission level we de- 
duce the hyperfine state of the atom as described above. The typical duration of one experimental cycle is $35 \mathrm{~ms}$, and it is repeated forty times for one atom. This leads to a high information rate exceeding typical rates when the atomic state is measured with push-out techniques [18. Due to thermal oscillations and varying position, the atom experiences different coupling strengths. Recording the transmission level for $10 \mathrm{~ms}$ with the repumping laser switched on at the end of each measurement cycle, we post select only those events with a strong atom-cavity coupling, i.e. where the transmission level lies below $30 \%$ of the empty cavity transmission before and after probing the normal mode splitting.

The population in $F=3$ as a function of the detuning $\omega_{\mathrm{p}}-\omega_{\mathrm{c}}$ is shown in Fig. 3, revealing the interaction-induced normal-mode splitting. Here, the cavity-atom detuning is $\omega_{\mathrm{c}}-\omega_{4,4^{\prime}}=$ $2 \pi \times 10 \mathrm{MHz}$, extracted from the numerical model (see below), where $\omega_{4,4^{\prime}}$ is the frequency of the $F=4 \longrightarrow F^{\prime}=4$ transition. By performing the same sequence without the $70 \mu \mathrm{s}$ mapping pulse we detect a background of approximately $13 \%$ erroneous detection events of the atomic state during the $2 \mathrm{~ms}$ state detection time. This value is compatible with the probability of $18 \%$ of a quantum jump to occur during the detection interval.

We analyze our measurement with a simple model: First, we estimate the photon scattering rate of the atom as a function of the cavity-probe laser detuning by numerically solving the master equation [19]. With this scattering rate we employ a rate equation to model the nonlinear population transfer to the $F=3$ state during the $70 \mu$ s mapping pulse. For this we assume a three-level system comprised of the $F=3$ and $F=4$ ground states and the $F^{\prime}=4$ excited state, neglecting population redistribution over Zeeman sublevels. We assume a homogeneous distribution of $g=2 \pi \times$ (6 ..12) MHz, which corresponds to the selected range of transmissions mentioned above. Here, $g=2 \pi \times 12 \mathrm{MHz}$ belongs to a maximally coupled atom for the $F=4, m_{F}=4 \longrightarrow F^{\prime}=4, m_{F^{\prime}}=4$ transition. Fitting the model to the measured data yields as two fit parameters the empty cavity photon number $n_{\mathrm{ph}}=0.062(3)$ and the cavity-atom detuning $\omega_{\mathrm{c}}-\omega_{4,4^{\prime}}=2 \pi \times 10(1) \mathrm{MHz}$ (solid line in inset of Fig. 33. An independent photon number measurement agrees with the fitted value and implies that we are in the weak excitation limit.

Extending our experiment to the case of two atoms coupled to the resonator, each atom affects the light field in the resonator which is experienced by the other atom. The probability of a quantum jump to occur from $F=4$ to $F=3$ then de-

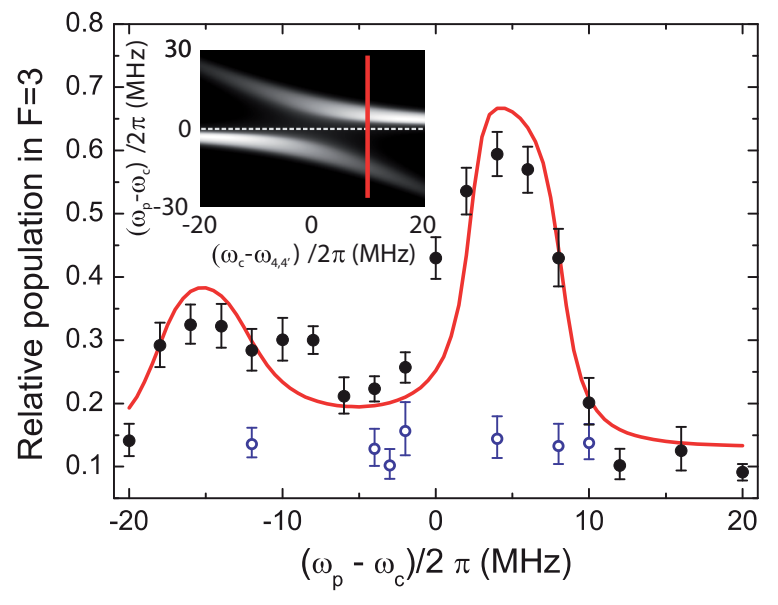

FIG. 3: Normal mode splitting, measured via population in $F=3$ versus probe-cavity detuning. Each data point (•) is the result of 120 to 470 experimental cycles. Erroneous detection of an atom in $F=3$ causes a background of $13 \%(O)$. Each such point is measured immediately after the data point for a certain detuning and therefore plotted at this detuning value. The solid curve is the result of our model (see text). The inset shows the calculated scattering rate of a single atom inside the cavity.

pends on the number of atoms in $F=4$. Thus, the dynamics of quantum jumps becomes conditional on the state of both atoms. While in our current experiment the coherent evolution of a quantum state due to this interaction cannot be observed due to photon scattering, the effective interaction does change the atomic spin dynamics.

We check that the distance between two atoms is below $2 \mu \mathrm{m}$, smaller than the waist of the cavity. We then position both atoms about $15 \mu \mathrm{m}$ away from the cavity axis, and pump them to $F=4$. There, for our parameters, the intra-cavity photon number depends sensitively on the number of atoms in $F=4[15,16]$. We monitor the probe laser transmission for about $120 \mathrm{~ms}$ while the repumping laser is switched off. Averaging over 169 of such traces we obtain the ensemble average shown in Fig. 4. This dynamics of the transmission signal is well explained without free parameters by assuming that the rate of quantum jumps depends on the state of both atoms. For a single atom in $F=4$, the rate of quantum jumps is measured independently to be $R_{1}=68 \pm 2 \mathrm{~s}^{-1}$ (see Fig. (4). When both atoms are in $F=4$, the quantum jump rate for each atom $R_{2}$ is extracted by comparing the initial transmission levels for one and two coupled atoms, yielding $R_{2}=28 \pm 1 \mathrm{~s}^{-1}$. Taking this into account, we obtain the theoretical transmission dependence depicted in Fig. 4. It agrees well with the measured data and confirms the coupled 


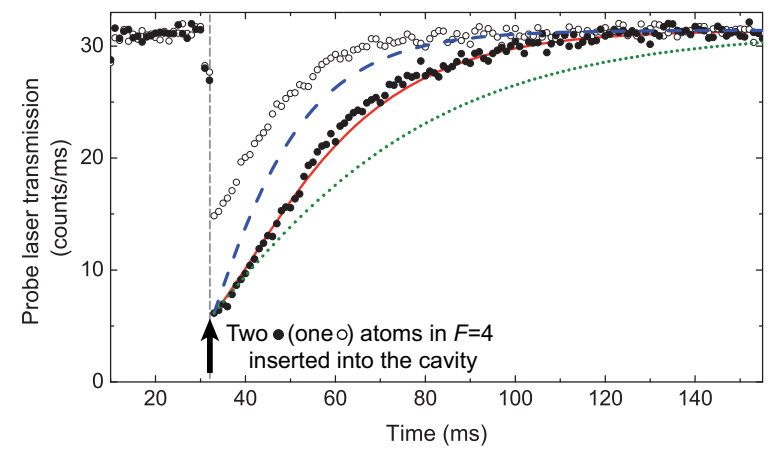

FIG. 4: Averaged cavity transmission for one (O) and two atoms $(\bullet)$ simultaneously coupled to the cavity. The solid line is the theoretical expectation for coupled dynamics of two atoms. For comparison, the dashed (dotted) line assumes an atom number independent rate of quantum jumps $R=R_{1}\left(R=R_{2}\right)$. The arrow indicates the insertion of atoms. The data has been taken with improved detection efficiency of $4.5 \%$ and a binning time of $1 \mathrm{~ms}$.

dynamics of atomic spins, while the assumption of an atom number independent rate yields an inconsistent behavior. Our signal is an evidence for an effective atom-atom interaction, where the measurement method relies on photon scattering thus objecting the creation of entangled states [6]. The non-linear dependence of the intra-cavity photon number on the number of atoms in $F=4$, however, is the basis for a conditional phase shift. The resulting dynamics can deterministically induce entanglement of the two atoms [21].

Summarizing, we have investigated the spin dynamics for one atom coupled to a high finesse resonator by non-destructively measuring the atomic state. Continuous probing the system by this detection method reveals quantum jumps of the atom. Further, we have measured the normal mode splitting of the strongly interacting atomcavity system via the atomic excitation. Finally, we have observed evidence for an effective atomatom interaction in spin dynamics of two atoms simultaneously coupled to the resonator mode. Our measurement method fulfills all requirements for a projective quantum non-demolition measurement of the state of a single atom [1, 22] on a timescale short compared to the inverse jump rate. Operating in a different regime, e.g. where the effect of atom-field interaction can be deduced from the phase shift of the transmitted probe light [2, 23, could improve the continuous measurement to the point required for active feedback onto the quantum state [24.

We acknowledge financial support by the EC (IP SCALA). SR acknowledges support from the "Deutsche Telekom Stiftung" and TK ac- knowledges support from the "Studienstiftung des deutschen Volkes".

* Electronic address: mika@iap.uni-bonn.de

[1] S. Haroche and J.-M. Raimond. Exploring the quantum. Oxford (2006).

[2] C.J. Hood et al. Science 287, 1447 (2000).

[3] P. W. H. Pinkse, T. Fischer, P. Maunz, and G. Rempe. Nature 404, 365 (2000).

[4] J. McKeever, J. R. Buck, A. D. Boozer, and H. J. Kimble. Phys. Rev. Lett. 93, 143601 (2004).

[5] S.-B. Zheng and G.-C. Guo. Phys. Rev. Lett. 85, 2392 (2000). L. You, X. X. Yi, and X. H. Su. Phys. Rev. A 67, 032308 (2003).

[6] A. S. Sørensen and K. Mølmer. Phys. Rev. Lett. 91, 097905 (2003).

[7] W. Nagourney, J. Sandberg, and H. Dehmelt. Phys. Rev. Lett. 56, 2797 (1986). Th. Sauter, W. Neuhauser, R. Blatt, and P. E. Toschek. Phys. Rev. Lett. 57, 1696 (1986). J. C. Bergquist, R. G. Hulet, W.M. Itano, and D. J. Wineland. Phys. Rev. Lett. 57, 1699 (1986).

[8] Th. Basche, S. Kummer, and C. Brauchle. Nature 373, 132 (1995).

[9] S. Peil and G. Gabrielse. Phys. Rev. Lett. 83, 1287 (1999).

[10] S. Gleyzes et al. Nature 446, 297 (2007).

[11] A.D. Boozer et al. Phys. Rev. Lett. 97, 083602 (2006).

[12] P. Maunz et al. Phys. Rev. Lett. 94, 033002 (2005).

[13] A. Boca et al. Phys. Rev. Lett. 93, 233603 (2004).

[14] For the atomic transition frequencies we have taken into account the AC Stark shift caused by the dipole trap.

[15] M. Khudaverdyan et al. New J. Phys. 10, 073023 (2008).

[16] For these parameters our system is in the weak excitation regime, i.e. the atomic excitation rate is much smaller than the atomic decay rate.

[17] R. J. Cook and H. J. Kimble. Phys. Rev. Lett. 54, 1023 (1985).

[18] D. Schrader et al. Phys. Rev. Lett. 93, 150501 (2004); J. Volz et al. Phys. Rev. Lett. 96, 030404 (2006); M. P. A. Jones et al. Phys. Rev. A 75, 040301 (2007).

[19] H. Carmichael An Open Systems Approach to Quantum Optics. Springer (1993))

[20] S. Osnaghi et al. Phys. Rev. Lett. 87, 037902 (2001).

[21] X.X. Yi, X.H. Su, and L. You Phys. Rev. Lett. 90, 097902 (2003). G.-W. Lin et al. Phys. Rev. A 77, 032308 (2008).

[22] V. Braginsky, Y. Vorontsov, and K. Thorne. Science 209, 547 (1980).

[23] P. J. Windpassinger et al. Phys. Rev. Lett. 100, 103601 (2008).

[24] A. R. R. Carvalho and J. J. Hope. Phys. Rev. A 76, 010301 (2007). A.R.R. Carvalho, A. J. S. Reid, and J. J. Hope. Phys. Rev. A 78, 012334 (2008). 